Original Research Paper

\title{
Development of New External Chest Compression Robotic System for Cardiopulmonary Resuscitation Using the Principles of Theranostics
}

\author{
${ }^{1}$ Nikolai A. Gryaznov, ${ }^{1}$ Konstantin Yu. Senchik, ${ }^{1,2}$ Vyacheslav V. Kharlamov and ${ }^{1}$ Galina S. Kireeva \\ ${ }^{1}$ Russian State Scientific Center for Robotics and Technical Cybernetics, St. Petersburg, Russia \\ ${ }^{2}$ Laser Technology Center, St. Petersburg, Russia
}

\author{
Article history \\ Received: 09-11-2015 \\ Revised: 13-11-2015 \\ Accepted: 05-03-2016 \\ Corresponding Author: \\ Vyacheslav V. Kharlamov \\ Russian State Scientific Center \\ for Robotics and Technical \\ Cybernetics, St. Petersburg, \\ Russia \\ Email: v.harlamov@rtc.ru
}

\begin{abstract}
The article deals with theoretical approaches to the design of the experimental samples of robotic outer chest compression system for Cardiopulmonary Resuscitation (CPR) functioning in accordance with principles of theranostics. The required number of components and their characteristics has been determined and justified in order to realize the mentioned principles in the robotic system. The software to control the operation of the experimental sample of the robotic system has been developed. This article presents the first data on the development of the new external cardiac compressor working in the adaptive mode and describes electronics used in this compressor.
\end{abstract}

Keywords: Cardiopulmonary Resuscitation (CPR), External Cardiac Compressor (ECC), Robotic System, Theranostics

\section{Introduction}

Fast evolution of electronics, computer technologies, physical element base, introduction of new materials with unique properties, increase in computer power has made it possible to create a new class of medical robotic systems incorporating diagnostic and therapeutic capabilities. A new scientific field combining the processes of diagnostics and therapy has been developed which is called theranostics. This article presents an example of practical application of theranostics principles creating a new medical device with functional feedback to perform Cardiopulmonary Resuscitation (CPR)- the External Cardiac Compressor (ECC).

\section{Theoretical Background and Literature Review}

$\mathrm{CPR}$ is a medical procedure performed in patients for maintaining blood circulation and respiratory function at a certain level when a patient's circulatory and respiratory functions are limited or do not exist at all. According to the statistics, only $5 \%$ of patients survive after CPR which is mainly due to low efficacy of manual chest compressions not allowing to improve patient's blood pressure (up to $90 \mathrm{mmHg}$ ) (Plaisance et al., 1999). To increase the CPR effectiveness special devices are used, such as defibrillators (increasing the effectiveness of CPR during manual chest compressions) and automatic devices for pneumatic auxiliary compression. Intellectual mechatronic feedback ECC were developed over the past 10-15 years. The main patent holders in this area are such companies as ZOLL (USA) and PhysioControl (USA) (Ong et al., 2006; Zheng et al., 2001; Becker et al., 1993; Eisenberg et al., 1990; Ornato et al., 2003; Rubertsson et al., 2007).

Clinical experience of application of existing cardiac compressors has revealed the need for improving their functional characteristics (Rubertsson et al., 2014; Steen et al., 2003; Sato et al., 1997; Paradis et al., 1990; Eftestøl et al., 2002; Rubertsson et al., 1995; Smekal et al., 2009; Wagner et al., 2010; Webb et al., 2002; Noc et al., 2007; Steen et al., 2002; Rubertsson and Karlsten, 2005), particularly with regard to the adaptive parameters changings depending on the value of the blood pressure, which is an integral index of the degree of blood circulation in patient during CPR (Malzer et al., 1996; Rich et al., 1981; Smith et al., 1990).

New mechatronic automated ECC has been developed and successfully tested in the Russian State Scientific Center for Robotics and Technical Cybernetics (Saint-Petersburg, Russia). This ECC has a feedback from the force applied to the chest and the striker position (which is in direct contact with the 
patient's chest). There are also algorithmically supposed feedbacks from blood pressure sensors with following changes in compression parameters, which enables automatic diagnostics of cardiovascular system functioning and the adjustment of ECC functional modes, i.e., allows applying principles of theranostics in practice.

\section{Methods and Tools}

\section{Software for the Automated External Cardiac Compressor}

The software for ECC is designed to ensure that the component parts of the device are operating according to the preset algorithm.

The ECC includes:

- Electric drive with transmission, which converts the rotary motion of the motor into linear motion of the loading platform of the cardiac compressor

- Set of sensors to monitor the patient's condition, compression process and to operate the ECC (invasive pressure sensor, noninvasive pressure sensor, sensor of the force applied to the chest, sensor of the value of compression);
- Microcontroller control system with a set of interfaces, including one for additional sensors and systems. Connection interface (protocol converter) for additional equipment provided with galvanic isolation

- Additional sensors and systems: Doppler flow velocity meter, cardiograph, pulse oximetry sensor, body temperature sensor, respiratory rate detection system, etc. (up to four sensors simultaneously) to monitor the patient's condition with the possibility to include these sensors in the process of the ECC monitoring and operation (additional equipment must provide their own primary power)

- Indication device (graphic display)

- Input device (keyboard)

- Warning device (buzzer, light-emitting diode)

- A secondary power supply for generating power to the system components

- System consisting of a set of compression sleeves for limbs with electronically controlled pneumatic pump unit and pressure sensors, carrying out the measurement of pressure oscillographically

- Autonomous power supply (lithium battery)

The developed ECC is presented on Fig. 1.

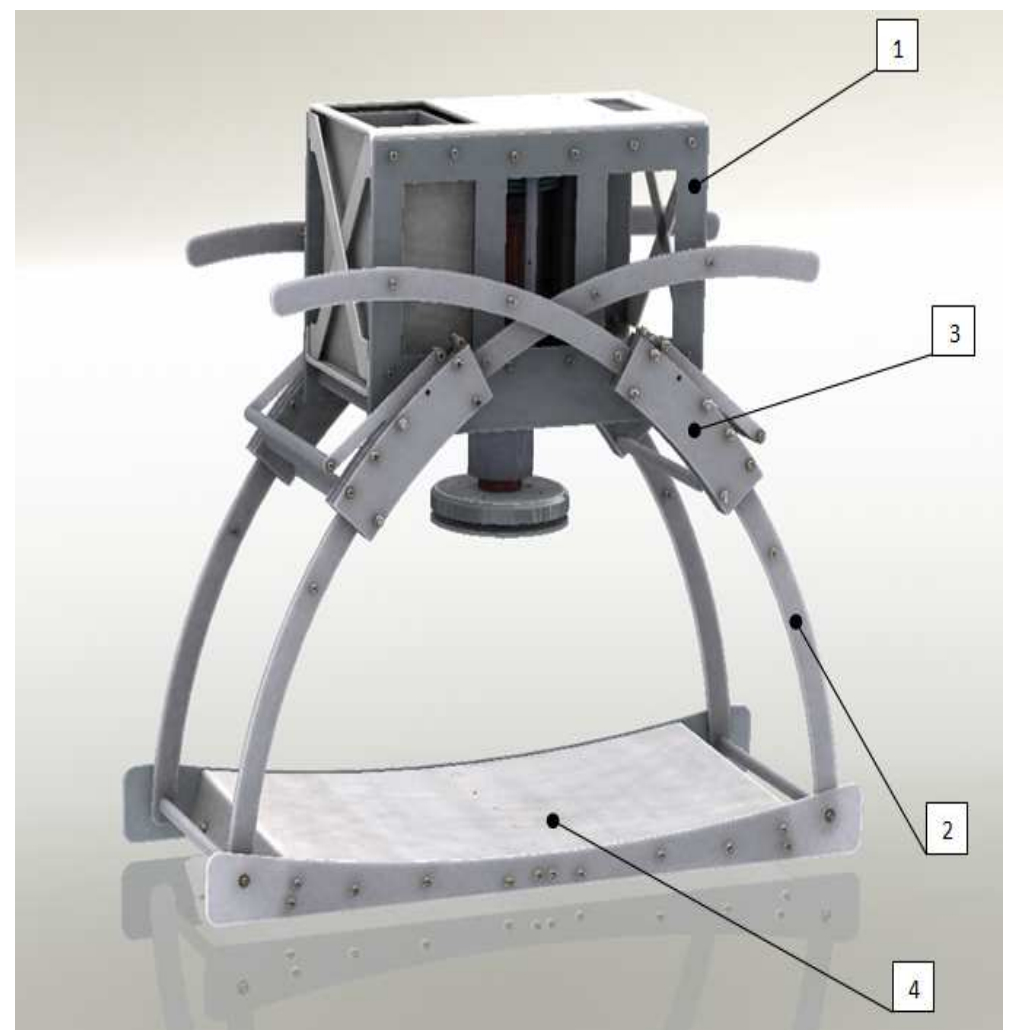

Fig. 1. General appearance of external cardiac compressor: mechatronic drive (1), rod attachment (2), position control mechanism for the actuator depending on the size of the patient's thorax (3), dorsal plate (bottom) (4) 
Both construction of the device and the software should firstly provide extremely fast connecting of the ECC to the patient in all kinds of situations with minimal time lag in resuscitation as well as the diagnostics of major cardiovascular system parameters of the patient. That is why all the preparatory operations (setup, calibration, parameter setting, etc.) must be carried out prior to install the device on the patient. Adjustment of the parameters and ECC modes witching must be carried out with minimal manipulation. Adjusting the optimum rate and depth of compression is very important, as these values have individual features and exert a strong influence on the performance of the compression process. It is known that the compression rate has a greater impact than the amount of the chest deformation (the depth of compression).

As long as ECC can be equipped with many diagnostic devices it can also be used to monitor vital parameters of the patient without performing compression cycles. Such division implies four major operation modes of ECC:

- "Diagnostics"- determining the patient's vital signs without performing chest compressions

- "Set-up"- pre-setting and operational testing prior to application

- "Operation"- the intended use

The latter mode in its turn includes the following modes:

- "Basic"-working with pre-configured parameters recommended by the internal sensors

- "Advanced"- work with the built-in connection system and additional sensors according to pretested algorithms. This mode should be expanded with the experience of the device operation gained

The ergonomics of the ECC electronic part must also comply with the functions it performs, which defines such basic requirements as:

- The fastest as possible positioning of ECC on the patient's chest to minimize the time lag in resuscitation and 'one-touch' actuation with the preset parameters and the minimal number of sensors and systems

- The possibility of subsequent 'hot' connection of sensors and systems in the working process with a serial input of them into the process of monitoring and control

- A convenient top location and good protection of the device controls for fast setting and adjusting the compression parameters
- convenient top position of the display, ergonomic screens corresponding with the current mode of operation and containing only the necessary information and, where possible, instructions (tips)

- multistage control of overshoots options with distinct alarm (light and sound)

Since ECC is not a precision instrument, the requirements for measurement accuracy can thus be reduced. Based on the above requirements, it is possible to determine an adequate set of system components and their basic characteristics, which in turn determines the type and number of software interfaces and control routines for data collection.

\section{Selection of the Components Ensuring Functioning of the Automated External Cardiac Compressor as a Theranostic Device}

The ECC includes the following sensors:

- A basic movement sensor for detecting the loading platform position (mm, accuracy of $\pm 1 \mathrm{~mm}$ )

- An acceleration sensor located at the loading platform for the secondary motion control (the loading platform position, $\mathrm{mm}$, accuracy of \pm 3 $\mathrm{mm}$ ) and for the compression process dynamics record. Zero points are given for a zero acceleration of the platform that is detected by the acceleration sensor

- A pressure sensor in the loading platform to record the load on the chest by a drive (in $\mathrm{kgf}$, accuracy $\pm 1 \mathrm{kgf}$ )

- An invasive blood pressure sensor for the further control of ECC functioning and operative control of the patient's condition (in $\mathrm{mmHg}$, accuracy of $\pm 5 \mathrm{mmHg}$ ). This sensor can also be used for automatic tuning parameters impact on the patient's chest (the depth of compression force) with the changes in his status

- Anon-invasive blood pressure sensor is connected to a system of controlled pneumatic cuffs with periodic measurement of the blood pressure parameters (in $\mathrm{mmHg}$, accuracy of $\pm 10 \mathrm{mmHg}$, measurement frequency of $1 \mathrm{~min}$.) and checking the presence of pressure pulsations in the cuff. To squeeze the limbs and pulsation measurements the sensor is connected permanently and the pressure is measured only when the additional sensor is connected through the interface

It is necessary to emphasize a special importance of applying the electrocardiograph during resuscitation, which makes it possible not only to reliably determine the restoration of cardiac activity, but, if necessary, to 
synchronize the operation of the ECC with the restored heart rhythm.

\section{Selection of the Power Supply}

Lithium iron phosphate battery was selected as a source of autonomous power. This type of battery was created by NASA program specifically to work in difficult conditions (Nishimura et al., 2010). For the ECC two series-connected batteries $4 \mathrm{~S}(12 \mathrm{~V}+12 \mathrm{~V})$ with a capacity of 4.2 A-h each are used. The batteries have two connectors- for charging and energizing, enabling to use them with enough power charger as an uninterruptible power supply. In the standalone mode, the batteries provide the ECC systems with an average power consumption of $80 \mathrm{~W}$ per $1 \mathrm{~h}$.

Functional Scheme of the Automated External Cardiac Compressor and its Interface Built in Accordance with the Theranostics Principles

Out of the entire functional scheme a subsystem for the compression parameters correction in accordance with the measured integral blood pressure can be singled out. Actuation of this subsystem converts the executive mechanism into both medical and diagnostic (theranostic) apparatus. The system (subsystem) of compression control according to the integral pressure includes invasive and noninvasive (with a set of cuffs and a pneumatic pump unit) pressure sensors, hardware and software for controlling the measurement of current pressure values and calculating the integral pressure control parameters for a given criterion. The functional scheme of the subsystem is presented in Fig. 2.

The subsystem also includes warning system receiving signals from additional external sensors. The elements not included in the system are represented by a dotted line. The integral pressure is understood by the authors as:

- The average pressure in 4 cuffs imposed on a limb: These measurements are carried out at $1 \mathrm{~min}$ intervals using oscillographic method

- The average pressure over the period of compression or a heartbeat in case of restored cardiac activity: Fast measurements using invasive pressure sensor with the sampling frequency of the sensor in the range of $1-10 \mathrm{kHz}$

The average pressure characterizes the energy of directed blood motion and is calculated as a sum of the diastolic (lower) and 1/3 pulse pressure.

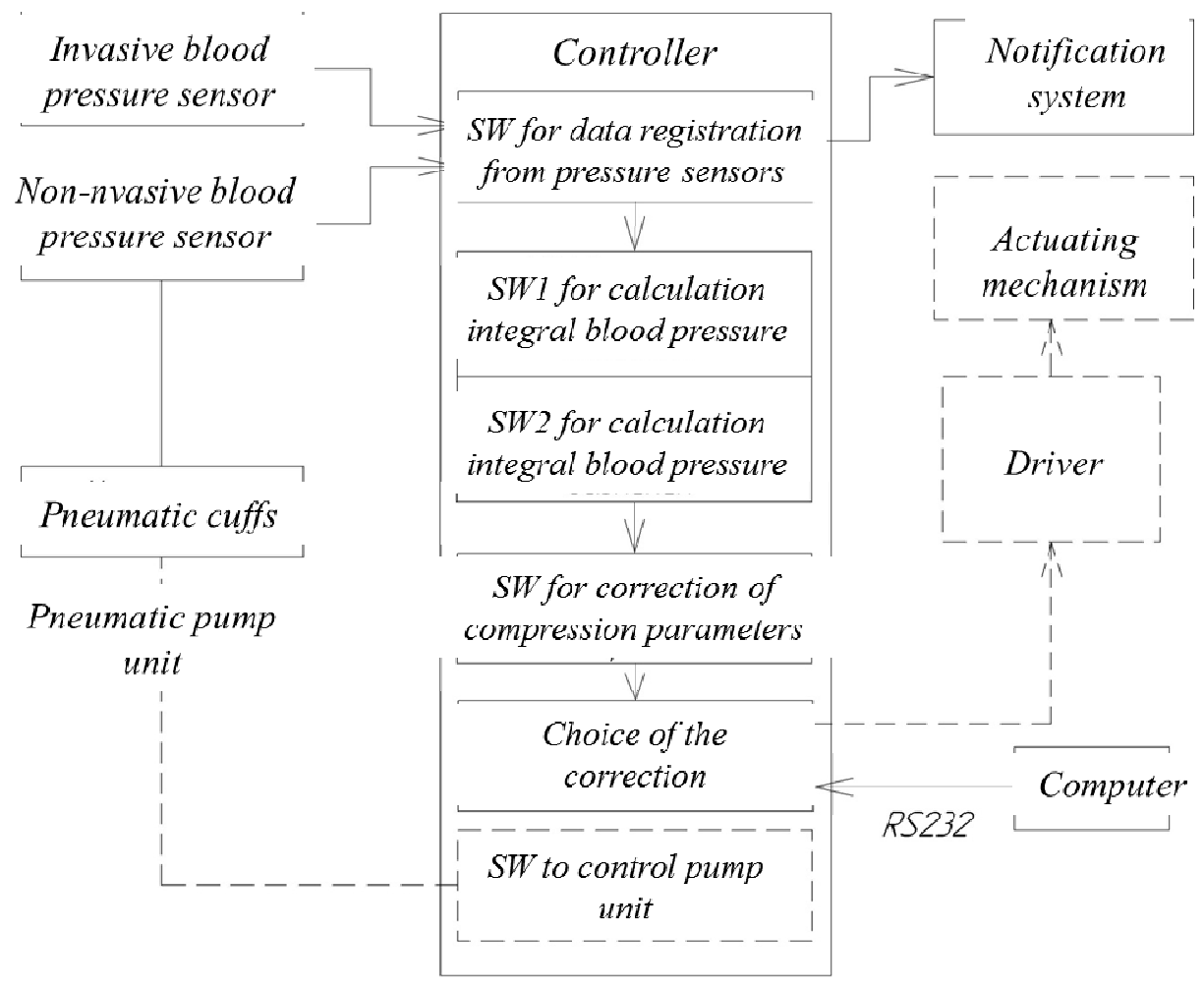

Fig. 2. System of compression control in accordance with the values of integral blood pressure 
Pulse pressure, in turn, represents the difference between the systolic (the top number) and diastolic (the bottom number) pressures. Also the presence of additional low pressure pulsations in the limbs pinched with cuffs (indirect check availability rate) is recorded.

\section{Results}

As a result of research the choice of components for the implementation of the principles theranostics was defined and justified. 3D model of external cardiac compressor was developed. On the basis of the data an experimental prototype robotic system has been assembled and software has been developed. The external cardiac compressor has a force feedback to control the process. This provides additional information for the doctor. In addition, the device has a feedback of arterial pressure, which suggests an adaptive mode of operation. This enables automated diagnostics of the cardiovascular system functioning, which allows applying principles of theranostics in practice.

\section{Discussion}

This article presents data on the development of a new external ECC. It has a blood pressure measurement system to adjust the pump unit. This system allows accelerating the process of data processing with regard to the state of the cardiovascular system, to make this information more accessible and optimize the setup algorithm. It allows realizing an adaptive mode. We suppose that it will improve the outcomes of patients after the cardiopulmonary resuscitation procedure. However, further clinical trials are essential to implement this ECC in healthcare organizations.

\section{Conclusion and Further Research}

The effectiveness of the integration of the automated external cardiac compressor built on the principles of theranostics into the modern medical process can be achieved by incorporating it as a part of a more complex system, but in this case theranostic features of this larger system should be considered.

\section{Acknowledgement}

The authors would like to express their gratitude to the management of the Central Research Institute of Robotics and Cybernetics for their support.

\section{Funding Information}

This article was prepared with financial support from the Ministry of Education and Science of Russian Federation for the research under the Agreement of
17.06.2014, grant 14.575.21.0014 (unique identifier RFMEFI57514X0014) for the implementation of the federal target program "Research and development on priority directions of scientific-technological complex of Russia for 2014-2020 years".

\section{Author's Contributions}

Nikolai A. Gryaznov: Organized the study, information checking and manuscript writing

Konstantin Yu. Senchik: He was engaged in the results analysis, drafting of article.

Vyacheslav V. Kharlamov: He was responsible for data collecting and analysis of the results.

Galina S. Kireeva: Contributed in data collecting, writing and editing of the manuscript.

\section{Ethics}

The authors have no conflicts of interest in the development of the research and publication of this article.

\section{References}

Becker, L.B., D.W. Smith and K.V. Rhodes, 1993. Incidence of cardiac arrest: A neglected factor in evaluating survival rates. Ann. Emerg. Med., 22: 86-91. DOI: 10.1016/S0196-0644(05)80257-4

Eftestøl, T., K. Sunde and P.A. Steen, 2002. Effects of interrupting precordial compressions on the calculated probability of defibrillation success during out-of-hospital cardiac arrest. Circulation, 105: 2270-2273. DOI: 10.1161/01.CIR.0000016362.42586.FE

Eisenberg, M.S., B.T. Horwood, R.O. Cummins, R. Reynolds-Haertle and T.R. Hearne, 1990. Cardiac arrest and resuscitation: A tale of 29 cities. Ann. Emerg. Med., 19: 179-186. DOI: $10.1016 / \mathrm{S} 0196-0644(05) 81805-0$

Malzer, R., A. Zeiner, M. Binder, H. Domanovits and G. Knappitsch et al., 1996. Hemodynamic effects of active compression-decompession after prolonged CPR. Resuscitation, 31: 243-253. DOI: 10.1016/0300-9572(95)00934-5

Nishimura, S., M. Nakamura, R. Natsui and A. Yamada, 2010. New lithium iron pyrophosphate as $3.5 \mathrm{~V}$ class cathode material for lithium ion battery. J. Am. Chem. Soc., 132: 13596-13597. DOI: 10.1021/ja106297a

Noc, M., B. Benz and K.B. Kern, 2007. Percutaneous Coronary Intervention (PCI) After Successful Reestablishment of Spontaneous Circulation and During Cardiopulmonary Resuscitation. In: Cardiac Arrest, the Science and Practice of Resuscitative Medicine, Paradis, N.A., H.R. Halperin, K.B. Kern, V. Wenzel and D.A. Chamberlain (Eds.), Cambridge University Press, New York, pp: 764-771. 
Ong, M.E., J.P. Ornato, D.P. Edwards, H.S. Dhindsa and A.M. Best et al., 2006. Use of an automated, load-distributing band chest compression device for out-of-hospital cardiac arrest resuscitation. JAMA, 295: 2629-2637.

DOI: $10.1001 /$ jama.295.22.2629

Ornato, J.P., M.A. McBurnie, G. Nichol, M. Salive and M. Weisfeldt et al., 2003. The Public Access Defibrillation (PAD) trial: Study design and rationale. Resuscitation, 56: 135-147. DOI: $10.1016 / \mathrm{S} 0300-9572(02) 00442-2$

Paradis, N.A., G.B. Martin, E.P. Rivers, M.G. Goetting and T.J. Appleton et al., 1990. Coronary perfusion pressure and the return of spontaneous circulation in human cardiopulmonary resuscitation. JAMA, 263: 1106-1113. DOI: 10.1001/jama.1990.03440080084029

Plaisance, P., K.G. Lurie, E. Vicaut, F. Adnet and J.L. Petit et al., 1999. A comparison of standard cardiopulmonary resuscitation and active compression-decompression resuscitation for out-ofhospital cardiac arrest. N Engl. J. Med., 341: 569-575. DOI: 10.1056/NEJM199908193410804

Rich, S., H.L. Wix and E.P. Shapiro, 1981. Clinical assessment of heart chamber size and valve motion during cardiopulmonary resuscitation by twodimensional echocardiography. Am. Heart J., 102: 368-373. DOI: 10.1016/0002-8703(81)90311-2

Rubertsson, S., A. Grenvik, V. Zemgulis and L. Wiklund, 1995. Systemic perfusion pressure and blood flow before and after administration of epinephrine during experimental cardiopulmonary resuscitation. Crit. Care Med., 23: 1984-1996. DOI: 10.1097/00003246-199512000-00007

Rubertsson, S., T. Huzevka, D. Smekal and J. Johansson, 2007. Early survival after cardiac arrest in a pilot study using the LUCAS device compared to manual chest compressions during CPR. Circulation, 116: 386-386.

Rubertsson, S. and R. Karlsten, 2005. Increased cortical cerebral blood flow with LUCAS; a new device for mechanical chest compressions compared to standard external compressions during experimental cardiopulmonary resuscitation. Resuscitation, 65: 357-363. DOI: $10.1016 /$ j.resuscitation.2004.12.006

Rubertsson, S., E. Lindgren, D. Smekal, O. Östlund and J. Silfverstolpe et al., 2014. Mechanical chest compressions and simultaneous defibrillation Vs conventional cardiopulmonary resuscitation in outof-hospital cardiac arrest: The LINC randomized trial. JAMA, 311: 53-61.

DOI: $10.1001 /$ jama.2013.282538
Sato, Y., M.H. Weil, S. Sun, W. Tang and J. Xie et al., 1997. Adverse effects of interrupting precordial compression during cardiopulmonary resuscitation. Crit. Care Med., 25: 733-736. DOI: 10.1097/00003246-199705000-00005

Smekal, D., J. Johansson, T. Huzevka and S. Rubertsson, 2009. No difference in autopsy detected injuries in cardiac arrest patients treated with manual chest compressions compared with mechanical compressions with the LUCAS device ${ }^{\mathrm{TM}}$ device-a pilot study. Resuscitation, 80: 1104-1107. DOI: 10.1016/j.resuscitation.2009.06.010

Smith, A.C., F.G. Spinale and M.M. Swindle, 1990. Cardiac function and morphology of Hanford miniature swine and Yucatan miniature and micro swine. Lab. Anim. Sci., 40: 47-50. PMID: 2153859

Steen, S., Q. Liao, L. Pierre, A. Paskevicius and T. Sjöberg, 2003. The critical importance of minimal delay between chest compressions and subsequent defibrillation: A haemodynamic explanation. Resuscitation, 58: 249-258. DOI: 10.1016/S0300-9572(03)00265-X

Steen, S., Q. Liao, L. Pierre, A. Paskevicius, T. Sjoberg, 2002. Evaluation of LUCAS, a new device for automatic mechanical compression and active decompression resuscitation. Resuscitation, 55: 285-299. DOI: 10.1016/S0300-9572(02)00271-X

Wagner, H., C.J. Terkelsen, H. Friberg, J. Harnek and K. Kern et al., 2010. Cardiac arrest in the catheterisation laboratory: A 5-year experience of using mechanical chest compressions to facilitate PCI during prolonged resuscitation efforts. Resuscitation, 81: 383-387.

DOI: $10.1016 /$ j.resuscitation.2009.11.006

Webb, J.G., N.K. Solankhi, S.K. Chugh, H. Amin and C.E. Buller et al., 2002. Incidence, correlates and outcome of cardiac arrest associated with percutaneous coronary intervention. Am. J. Cardiol., 90: 1252-1254.

DOI: 10.1016/S0002-9149(02)02846-1

Zheng, Z.J., J.B. Croft, W.H. Giles and G.A. Mensah, 2001. Clinical investigation and reports: Sudden cardiac death in the United States, 1989 to 1998. Circulation, 104: 2158-2163. DOI: $10.1161 /$ hc4301.098254 